\title{
Intrapelvic Nerve Entrapment Syndrome Caused by a Variation of the Intrapelvic Piriformis Muscle and Abnormal Varicose Vessels: A Case Report
}

\author{
Ahmet Kale ${ }^{1}$, Gulfem Basol ${ }^{1}$, Ahmet C. Topcu² Elif C. Gundogdu ${ }^{1}$, Taner Usta ${ }^{3}$, Recep Demirhan ${ }^{4}$ \\ ${ }^{1}$ Department of Obstetrics and Gynecology, University of Health Science Kartal Dr. Lutfi Kirdar City Hospital, Istanbul, Turkey \\ ${ }^{2}$ Department of Cardiovascular Surgery, University of Health Science Kartal Dr. Lütfi Kurdar City Hospital, Istanbul, Turkey \\ ${ }^{3}$ Department of Obstetrics and Gynecology, Acibadem University, Altunizade Hospital, Istanbul, Turkey \\ ${ }^{4}$ Department of Thoracic Surgery, University of Health Science Kartal Dr. Lutfi Kirdar City Hospital, Istanbul, Turkey
}

\begin{abstract}
Entrapment neuropathy of the sciatic nerve and pudendal nerve are painful syndromes that are often overlooked by physicians. Laparoscopic surgical interventions for nerve entrapment syndromes of the posterior pelvis focus on removing the compression lesion with the purpose of eliminating the suspected cause of sciatica, as well as pudendal neuralgia. Herein, the authors report the rare event of sciatic and pudendal nerve entrapment, which was caused by aberrant vessels and a variant piriformis muscle bundle, as a seldom-diagnosed cause of sciatica and pelvic pain, for both neurosurgeons and neuropelveologists. The authors present the laparoscopic decompression technique for the pudendal and sciatic nerves by giving our technical "tips and tricks" enriched by a surgical video.
\end{abstract}

Keywords: Laparoscopy; Neurosurgery for pain; Neurovascular entrapment; Piriformis muscle; Pudendal nerve; Sciatic nerve

- Research Ethics: This study was approved by the Kartal Dr. Lütfi Kurdar City Hospital's Institutional Review Board (IRB2020/514/79/36\#2020/06).

Informed consent for the collection and publication of data and video was obtained from the patient.

- Conflict of Interest: No potential conflict of interest relevant to this article was reported.

Entrapment neuropathy of the sciatic nerve and pudendal nerve are painful syndromes that are often overlooked by physicians. Predictably, if a nerve gets entrapped, symptoms are present based on the level of compression and radiate along the course of the nerve dermatome. The pain pattern, along with the presence or absence of accompanying symptoms, can guide the diagnosis. For instance, persistent pudendal neuralgia accompanied by sciatica might indicate nerve entrapment syndrome in the intrapelvic portion. Deeply infiltrating endometriosis, fibrotic tissue or vascular entrapment, and piriformis muscle syndrome are conditions responsible for the compres- sion of intrapelvic nerves that are often undiagnosed and are still considered rare [1].

Recent advances in laparoscopic surgery and the increasing interest in the concept of neuropelveology have allowed physicians to obtain diagnostic certainty prior to initiating treatment for many intrapelvic nerve pathologies. When intrapelvic nerve compression or irritation is highly suspected, intrapelvic nerve decompression should be considered as an etiologic treatment [2-5].

The first objective of this case report was to present a new perspective by discussing a case of sciatic and pudendal nerve

Corresponding author: Gulfem Basol (iD https://orcid.org/0000-0001-7738-8531 Department of Obstetrics and Gynecology, University of Health Science Kartal Dr. Lutfi Kirdar City Hospital, Cevizli Mah Tugay Yolu Cad. Nuvodragos Sitesi Maltepe, Istanbul, Turkey Email: glfem412010@hotmail.com

Submitted: June 29, 2020 / Accepted after revision: August 16, 2020 
entrapment, which was caused by aberrant vessels and a variant piriformis muscle bundle, as a seldom-diagnosed cause of sciatica and pelvic pain, for both neurosurgeons and neuropelveologists. The second aim was to present the laparoscopic decompression technique for the pudendal and sciatic nerves by giving our technical "tips and tricks."

\section{CASE REPORT}

A 42-year-old woman with a gravidity of 2 and parity of 2 presented with symptoms of severe, burning, sharp pain on the right sciatic and pudendal nerve dermatomes, which had been present for 8 years (visual analogue scale [VAS] pain score= $10 / 10)$, combined with urinary urgency and nocturia. She had not engaged in sexual activity for 2 years because of severe dyspareunia. Her pain was becoming unbearable with physical activity, prolonged standing, and in the sitting position. She could not walk for more than 30 minutes because of her pain, which had not responded to previous medical or physical treatment, pudendal nerve block therapy, or piriformis muscle injection.

On her neuropelveologic examination, hyperesthesia was observed in both the S2 and pudendal nerve dermatomes. Her muscle strength of the right lower extremity was assessed as grade 5/5 using the manual muscle testing method, and the neurologic examination was otherwise normal. A vaginal muscle palpation examination was performed in all 4 quadrants, and right obturator internus hypertonicity and tenderness were observed. The Tinel sign was positive on the right sacrospinous ligament. The painful piriformis muscle was palpated at the 7 or 8 oclock positions on a deep rectal examination. The Freiberg sign was positive on the right side. The seated piriformis stretch test and active piriformis tests were positive. Although magnetic resonance imaging (MRI) revealed dilated vessels on the right sciatic nerve (Fig. 1), it did not demonstrate any anatomical variation of the piriformis muscle. Furthermore, other neurogenic pathologies were excluded through MRI and a neuropelveologic examination. With a diagnosis of intrapelvic entrapment of the sacral nerve roots, the patient was recommended to undergo laparoscopic decompression surgery to treat the structures entrapping the sacral nerves causing pelvic pain, sciatica, and urinary symptoms.

The patient underwent standard preparations prior to the laparoscopic procedures. Upon laparoscopic exploration, the uterus and ovaries were seen to have been removed. The adhesions were released and the dissection area was reached. The

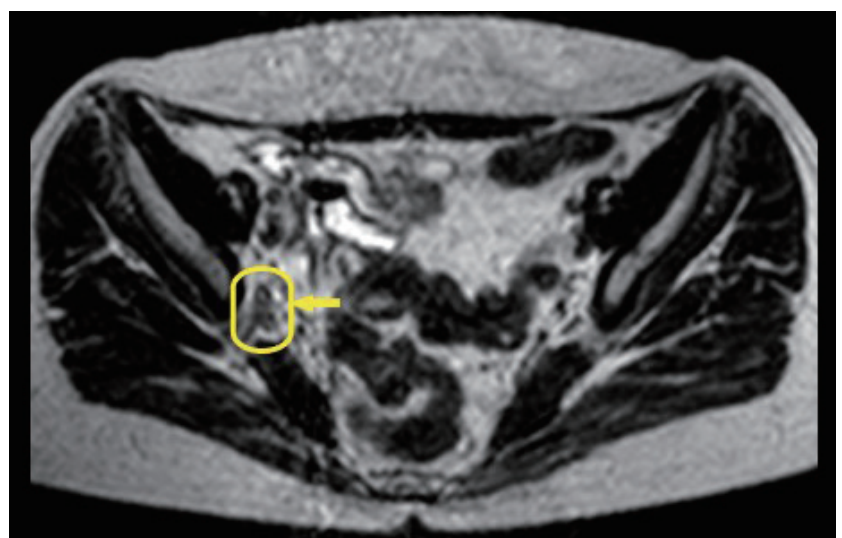

Fig. 1. Axial T2-weighted magnetic resonance image shows the aberrant vessel crossing over the sciatic nerve (arrow) anterior to the piriformis $(\mathrm{P})$ muscle.

first step was to create a wide peritoneal incision along the external iliac vessels toward the obliterated umbilical ligament on the anterior pelvic wall by using bipolar graspers and monopolar scissors. The dissection was deepened between the external iliac vessels and the medial edge of the iliopsoas muscle on the right side of the pelvis, as described previously [5]. The obturator nerve was identified in the right obturator fossa and gently mobilized medially. Multiple-branching superior gluteal vessels that were in contact with the lumbosacral trunk were reached at the depth of the iliolumbar fossa. These small, branching vessels were coagulated and cut using a LigaSure vessel-sealing device (Blunt Tip; Covidien, Dublin, Ireland). The dissection was continued caudally and multiple-branching enlarged vessels were seen, skeletonized, and separated from the sciatic nerve using the laparoscopic grasper (Richard Wolf GmbH, Knittlingen, Germany) and cold scissors.

The aberrant artery and veins, which were crossing over the sciatic nerve, were clipped using Hem-o-Lok clips (Weck Closure System; Teleflex, Wayne, PA, USA). The proximal and distal ends were released using the LigaSure device. After removing all aberrant vessels above the lumbosacral trunk, the sciatic nerve was visualized. On further dissection, the pelvic portions of the sciatic and pudendal nerve that were entrapped by the piriformis muscle bundle were identified. The piriformis muscle was gently dissected and separated from the surrounding tissue and the sciatic nerve. The muscle was suspended to prevent thermal spread to the sciatic nerve, and then the muscle was sealed and cut. The sacrospinous ligament was cut and the pudendal nerve was also released. At the end of the operation, the pudendal and sciatic nerves were seen to be completely 
freed (Supplementary video clip 1). The total surgical time was 110 minutes and the estimated blood loss was $50 \mathrm{~mL}$.

The patient was discharged from the hospital on the first postoperative day without any complications. One day later, just after the operation, her pain decreased dramatically and her pelvic pain and sciatica had completely resolved. At 3- and 6-month follow-up examinations, her VAS score was 0/0. The cutaneous allodynia and hyperesthesia along the S2 and pudendal nerve dermatome had completely resolved. Furthermore, urinary frequency and nocturia episodes resolved after 6 months. Informed consent for the collection and publication of data and video was obtained from the patient. This study was approved by the Kartal Dr. Lütfi Kurdar City Hospital's Institutional Review Board (IRB2020/514/79/36\#2020/06).

\section{DISCUSSION}

The term "sciatica" usually describes a symptom rather than a specific condition or disease. The mechanism of sciatic pain varies widely. Sciatic pain may be caused by many different mechanisms, including direct nerve compression (discogenic or nondiscogenic), inflammation, irritation, and trauma, or a combination of these conditions [6,7]. The search for intrapelvic pathologies for pain should be kept in mind when imaging techniques and examinations reveal no discogenic pathologies.

In the present case report, we found piriformis muscle entrapment accompanied by vascular entrapment of the sacral plexus. Beaton and Anson classified and demonstrated 6 different anatomic variations of the piriformis muscle and sciatic nerve. The piriformis muscle variant in our case was most consistent with type D in the Beaton classification, in which the sciatic nerve exited through the midsubstance of the piriformis muscle, with a reported proportion of $0.3 \%$ to $3 \%$ of cases [8]. What is less well known, however, is that enlarged or aberrant branches of the internal iliac vessels may cause sciatic pain as a result of compression or irritation of the pelvic nerves [3,4]. If conservative treatment is ineffective for symptom relief, and in the presence of high suspicion of intrapelvic nerve compression, surgery should be considered [5]. We noted that almost all patients described in the literature underwent the posterior approach for piriformis muscle release with a large incision by cutting the gluteus maximus muscle [9]. In contrast, Lemos and Possover [10] performed laparoscopic sacral plexus exploration for nerve decompression in a total of 213 patients. In their series, they reported isolated sacral plexus vascular entrapment in
37 patients and pyriformis syndrome in 1 patient. In our case, the patient's pain completely resolved, similar to the study by Lemos and Possover [10]. In our opinion, the surgical treatment of piriformis syndrome with the posterior approach is invasive and misses the possibility of identifying any possible coexisting pathology in the pelvic region.

Our laparoscopic surgical principle is based on conducting a full exploration of the lumbosacral trunk, sciatic nerve, and the pudendal nerve when intrapelvic nerve entrapment is suspected. Intraoperative and postoperative complications are possible in patients scheduled to undergo laparoscopic decompression of the pelvic nerves. The laparoscopic anterior approach for vascular decompression of the sacral plexus involves an additional surgical risk because of the diverse variations of pelvic vessels and nerves.

In this case report, we introduced our surgical techniques and principles based on reducing potential complications such as iatrogenic nerve injury and bleeding. The first tip is that meticulous dissection of the adjacent tissue around the lumbosacral trunk should proceed with smaller steps using bipolar cautery before cutting any tissue to promote the safety of neural tissues, because an aberrant and/or a variant vessel embedded in the adipose tissue may cause haemorrhage, which may disturb the laparoscopic vision. The second tip is that we prefer to transect vessels less than $5 \mathrm{~mm}$ in diameter and nonbranching vessels with a bipolar electrosurgical device. With relatively large-diameter and multiple-branching vessels, we apply Hemo-Lok clips prior to cutting them. We pay attention to several important principles when applying Hem-o-Lok clips. Before applying the clips, we perform a complete circumferential dissection of the vessels and separate them from the nerves, using a grasper and cold scissors. After that, we use an appropriately sized clip and place them at $90^{\circ}$ to the vessels, then we remove the applier carefully. To our knowledge, this case report is the first to describe laparoscopic intrapelvic nerve decompression surgery with an anterior approach in pelvic nerve entrapment syndrome caused by aberrant vessels and a variant piriformis muscle.

In cases of sciatic pain accompanied by pelvic and/or perineal pain and urinary symptoms, vascular entrapment accompanied by piriformis syndrome should be considered. Without question, in this condition, laparoscopic intrapelvic nerve decompression with the anterior approach should be preferred over the posterior approach for nerve release. However, laparoscopic treatment of intrapelvic entrapment requires a good 
knowledge of female pelvic neuroanatomy and advanced laparoscopic surgical skills based on neuropelveologic concepts.

\section{ACKNOWLEDGEMENTS}

Special thanks to Mr. David Chapman for his help with proofreading this manuscript.

\section{SUPPLEMENTARY MATERIAL}

Supplementary video clip 1 can be found via https://doi.org/ inj.2040232.116. Video clip 1. The video describes a case of the laparoscopic management of intrapelvic nerve entrapment, which was caused by aberrant vessels and a variant piriformis muscle bundle.

\section{ORCID}

$\begin{array}{ll}\text { Ahmet Kale } & 0000-0002-1194-6649 \\ \text { Gulfem Basol } & 0000-0001-7738-8531 \\ \text { Elif C. Gundogdu } & 0000-0003-2961-3831 \\ \text { Taner Usta } & 0000-0002-2208-3316 \\ \text { Recep Demirhan } & 0000-0003-4424-5918\end{array}$

\section{AUTHOR CONTRIBUTION STATEMENT}

- Conceptualization: $A K, G B, E G, R D$

- Data curation: $E G$

- Formal analysis: $T U, R D$

- Project administration: $A T, T U$

-Writing-original draft: $A K, G B, A T$

-Writing-review \& editing: $G B, A T, E G, R D$

\section{REFERENCES}

1. Possover M, Forman A. Pelvic neuralgias by neuro-vascular entrapment: anatomical findings in a series of 97 consecutive patients treated by laparoscopic nerve decompression. Pain Physician 2015; 18:E1139-43.

2. Possover M, Forman A. Neuropelveological assessment of neuropathic pelvic pain. Gynecol Surg 2014;11:139-44.

3. Possover M, Schneider T, Henle KP. Laparoscopic therapy for endometriosis and vascular entrapment of sacral plexus. Fertil Steril 2011;95:756-8.

4. Lemos N, Marques RM, Kamergorodsky G, Ploger C, Schor E, Girão MJBC. Vascular entrapment of the sciatic plexus causing catamenial sciatica and urinary symptoms. Int Urogynecol J 2016; 27:317-9.

5. Kale A, Basol G, Usta T, Cam I. Vascular entrapment of both the sciatic and pudendal nerve causing persistent sciatica and pudendal neuralgia. J Minim Invasive Gynecol 2018;26:360-1.

6. Al-Khodairy AWT, Bovay P, Gobelet C. Sciatica in the female patient: anatomical considerations, aetiology and review of the literature. Eur Spine J 2007;16:721-31.

7. Kulcu DG, Naderi S. Differential diagnosis of intraspinal and extraspinal non-discogenic sciatica. J Clin Neurosci 2008;15:1246-52.

8. Natsis K, Totlis T, Konstantinidis GA, Paraskevas G, Piagkou M, Koebke J. Anatomical variations between the sciatic nerve and the piriformis muscle: a contribution to surgical anatomy in piriformis syndrome. Surg Radiol Anat 2014;36:273-80.

9. Han SK, Kim YS, Kim TH, Kang SH. Surgical treatment of piriformis syndrome. Clin Orthop Surg 2017;9:136-44.

10. Lemos N, Possover M. Laparoscopic approach to intrapelvic nerve entrapments. J Hip Preserv Surg 2015; 2:92-8. 